\title{
Application of continuous wavelet transforms for swell noise attenuation
}

Gustavo Catão Alves*[1], André Bulcão[1], Bruno Pereira Dias ${ }^{[1]}$, Djalma Manoel Soares Filho[1] e Felipe Prado Loureiro[2]

[1] Petrobras; ${ }^{[2]}$ Fundação Gorceix

Copyright 2019, SBGf - Sociedade Brasileira de Geofísica

This paper was prepared for presentation during the $16^{\text {th }}$ International Congress of the Brazilian Geophysical Society held in Rio de Janeiro, Brazil, 19-22 August 2019.

Contents of this paper were reviewed by the Technical Committee of the $16^{\text {th }}$ International Congress of the Brazilian Geophysical Society and do not necessarily represent any position of the SBGf, its officers or members. Electronic reproduction or storage of any part of this paper for commercial purposes without the written consent of the Brazilian Geophysical Society is prohibited.

\begin{abstract}
Ocean-surface related noises known as swell noises are a common occurrence in marine surveys and present a challenge for traditional noise suppression techniques, due to the non-random nature of the events. In order to identify this type of noises, we can use differences between real and modeled data sets. This is particularly useful in the context of full waveform inversion, where both types of data are available. Here, we apply a continuous wavelet transform (CWT) to real and modeled data to attenuate swell noise events by estimating filters from the modeled data using a smooth velocity model and applying those to the real data. We show that filters using the CWT domain can efficiently attenuate swell noise by correctly estimating the time windows and wavelet scales of desired data.
\end{abstract}

\section{Introduction}

Noise attenuation is an important step in seismic data pre-processing, as it removes or at least diminishes the appearance of artifacts in migrated data (Yilmaz, 2001). For methodologies such as Full Waveform Migration (FWI), the importance of noise attenuation lies in the calculation of data residuals, as noise present in observed data can't be correctly modeled and will therefore show up in the inversion gradients (Virieux, 2009).

Swell noise is often observed as low frequency vertical streaks in marine data and is commonly observed in marine surveys during rough weather conditions. While swell noise attenuation in reflection imaging can be easily addressed by the application of a low-cut filter (Yilmaz, 2001), in FWI the solution becomes much harder, as low frequencies are an essential part of the inversion workflow (Virieux, 2009). Therefore, it is important to design filters that can attenuate coherent low frequency noises such as swell noise without affecting parts of the data needed by inversion schemes.

Wavelet transforms are a way to represent functions as a series of wavelets. Their use is widespread and can include image-processing methods based on discrete wavelet transforms (DWT), as well as design of biomedical designs and even wireless communications (Akansu, 2010). In this work, we propose the use of the continuous wavelet transform (CWT) as a means to achieve efficient localized frequency band filtering for swell noise attenuation.

\section{Method}

The first step in constructing filters based on wavelet transforms is the choice of a suitable set of wavelets and scales. We chose Morlet wavelets as the basis for the series we use for representing our seismic traces. Figure 1 shows the shape of these wavelets at different scalings.

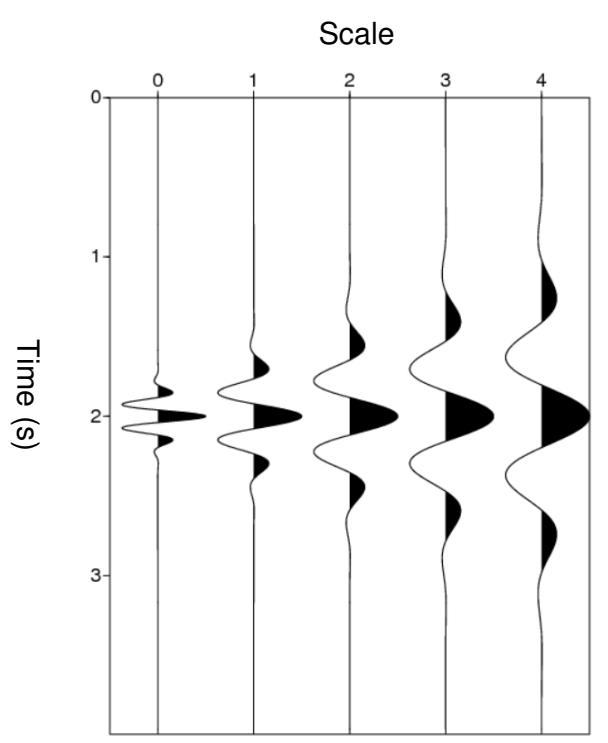

Figure 1 - Morlet wavelets at different scales.

Next, we apply the CWT to the seismogram, which means convolving each trace with the Morlet wavelet at many scales. This operation increases the dimensionality of the data set, as each seismic trace is now represented as a two-dimensional panel. In figure 2, we exemplify this by taking the CWT of two traces: one at near offset and another at far offset. We can see in the CWT of the near offset trace the transform related to the direct arrival, spanning several scales close to zero time. The primary shows up in the same panel at around 1 second. For the far offset, we see that the same primary is now evident at around 1.6 seconds, spanning the same scales as before.

We can apply the same procedure to both real and synthetic data sets. This allows us to produce CWT panels representing our "ideal" outputs and then create masks in the CWT domain to filter out undesired events in the real data set. In the next section, we explore this idea in more detail with a field example. 

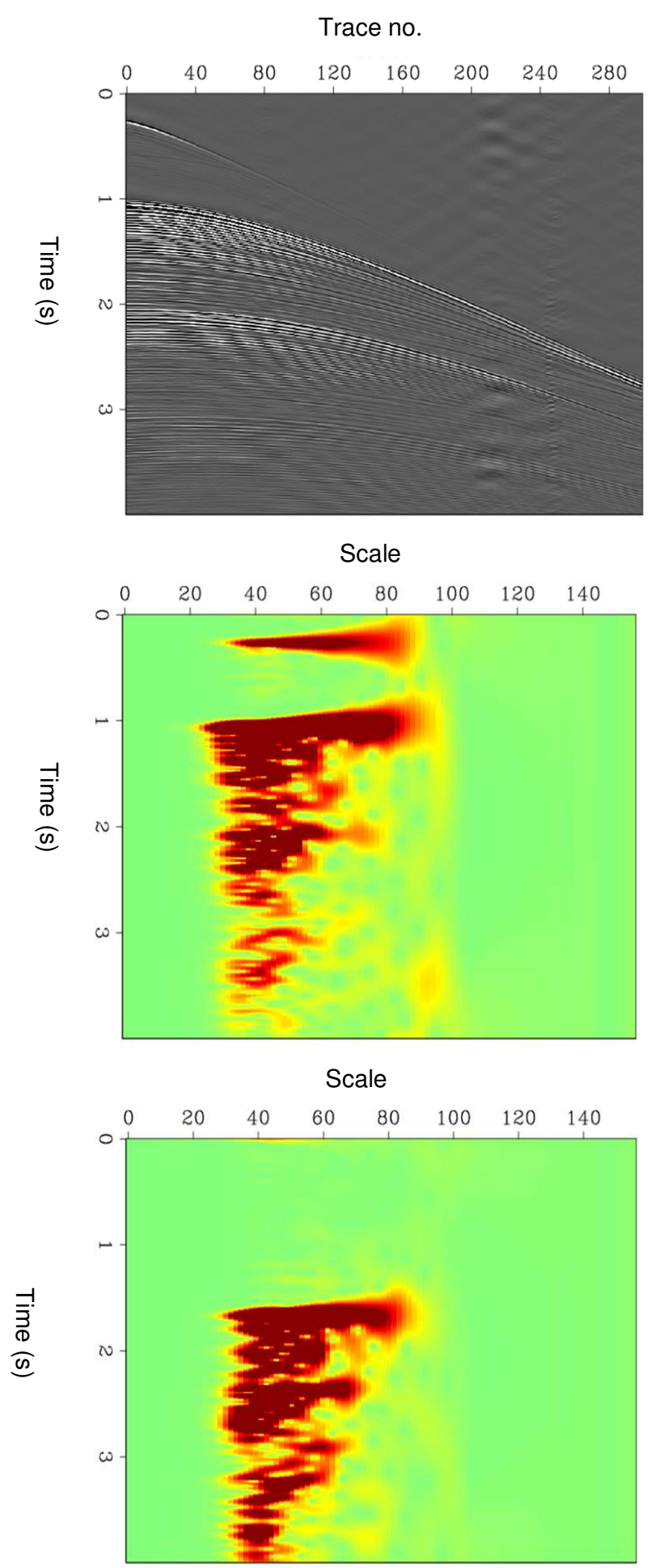

Figure 2 - Field data seismogram (top) and CWT transforms for a short offset trace (trace no. 1) (middle) and a far offset trace (trace no. 150) (bottom). CWT panels were normalized, with coefficients ranging from 0 (green) to 1 (red).

\section{Results}

Our field data comes from a marine streamer survey. We present data in common-shot gathers, with 638 traces per shot. For synthetic data, we use a finite difference numerical approximation of the acoustic wave equation. The velocity model employed for the simulations is a smooth model, the same used as an initial model for FWI.

In figure 4, we show a common-shot gather with the presence of swell noise in the far offset traces and the CWT associated to trace no. 5050, which is in the middle of the area contaminated by noise. Figure 5 shows the synthetic data for the same shot, together with the CWT for the same trace. We see that the synthetic shot gives us the overall shape of the clean trace in CWT space, so that we can define a filter to be applied to the real shot. We normalize values in all CWT panels, with coefficients ranging from 0 (green) to 1 (red).

We create the filter in CWT space by defining a threshold amplitude for coefficients at each scale in the synthetic data set. We zero the coefficients under this threshold, thus creating a mask around the expected data. We smooth the edges of the mask slightly, to avoid discontinuities in the inverse transform. For this particular example, the threshold was 0.4 or about $40 \%$ of the maximum values in CWT space.

Finally, we apply the resulting filter to the observed data. Figure 6 shows the result of applying a filter obtained from the simulated data in figure 5 to the real data from figure 4. It is evident that the filter efficiently attenuated the swell noise present in the gather, but without hard discontinuities created by muting in data space. It is also possible to notice that the filter even affected the noise overlapping the primary, resulting in a cleaner event.

\section{Conclusions}

We present a workflow for creating and applying a filter for coherent noise using continuous wavelet transforms.

Our results show that this method can efficiently attenuate swell noise present in the data, without the need for a low-cut frequency filter in Fourier space, which could affect low frequencies of useful data.

Our workflow is highly automated, needing only a threshold value to create the filter. This means that we can process a large data set with minimum user intervention. However, we must point out that results are much improved if we evaluate this threshold separately for noises at different offsets, as their shape in CWT space changes.

\section{Acknowledgments}

The authors would like to thank Petrobras for supporting this research and permission to publish this work. 
Trace no.
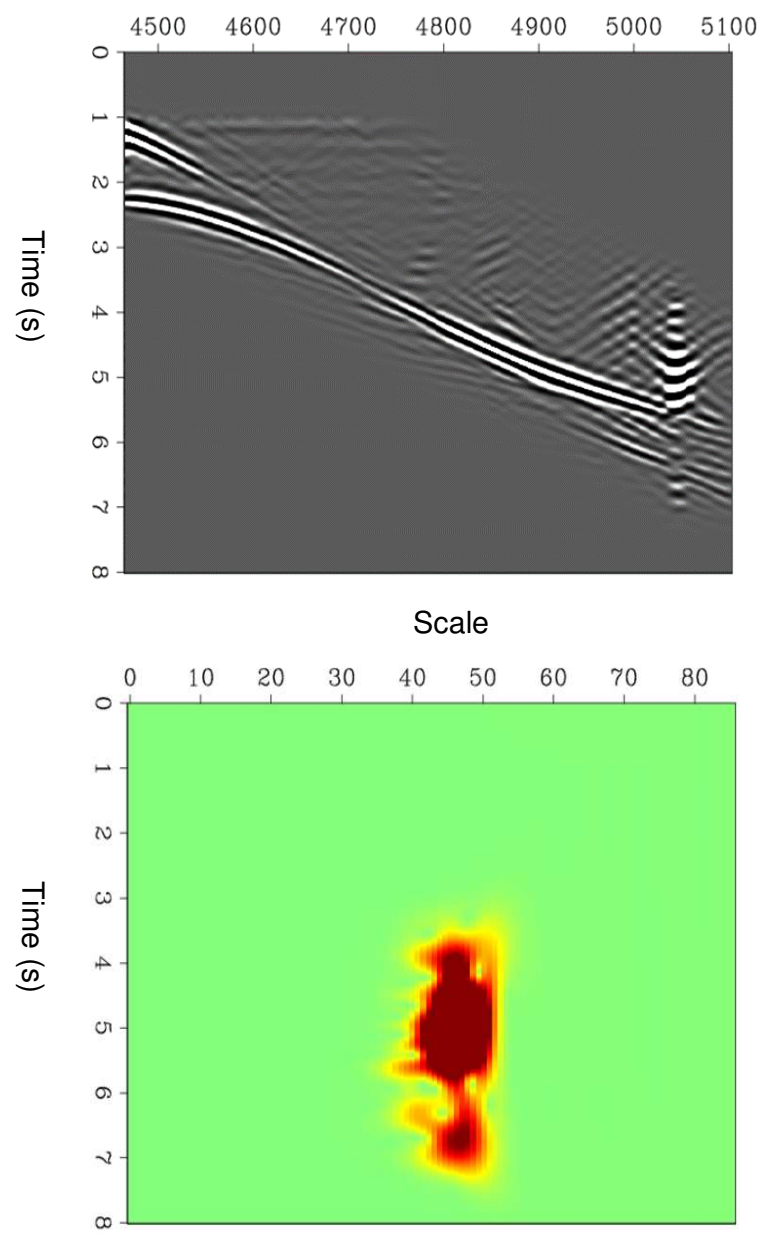

Figure 4 - Common-shot gather of a marine streamer survey showing the presence of swell noise at the far offsets (top); and the CWT panel of trace no. 5050 (bottom).
Trace no.

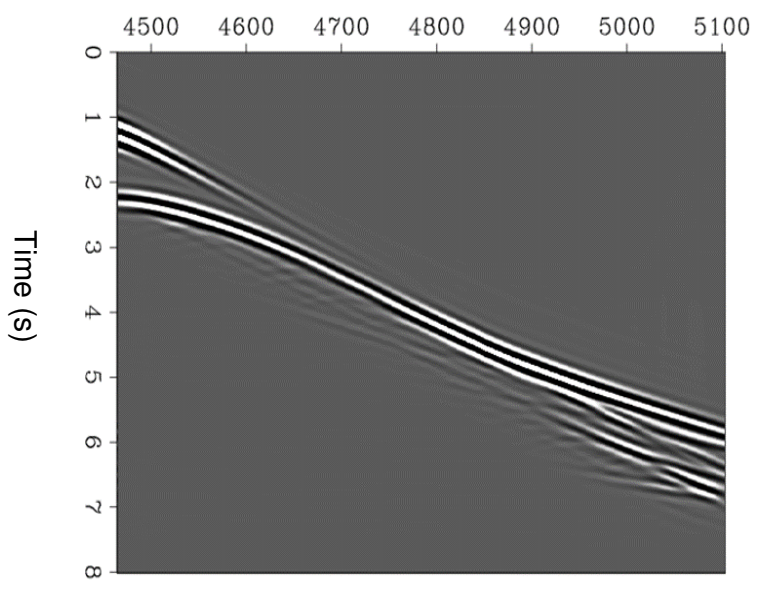

Scale

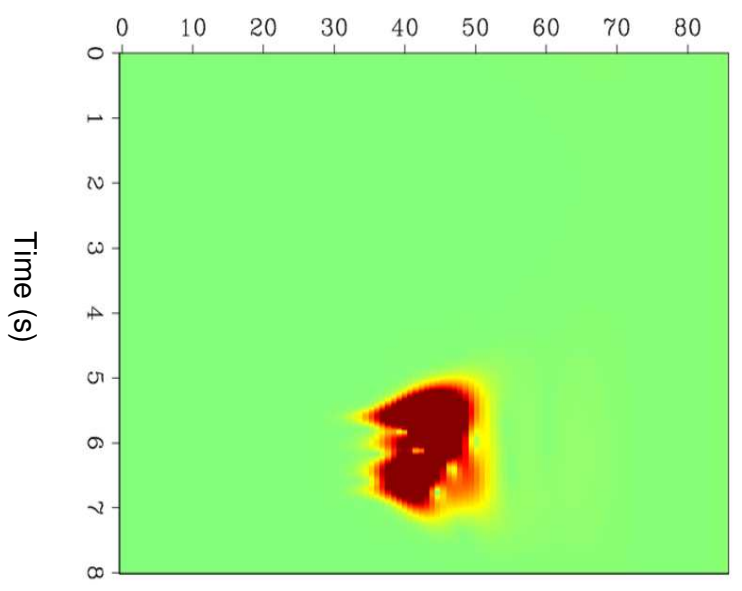

Figure 5 - Simulated common-shot gather of a marine streamer survey using a smooth velocity model (top); and the CWT panel of trace no. 5050 (bottom).

Trace no.

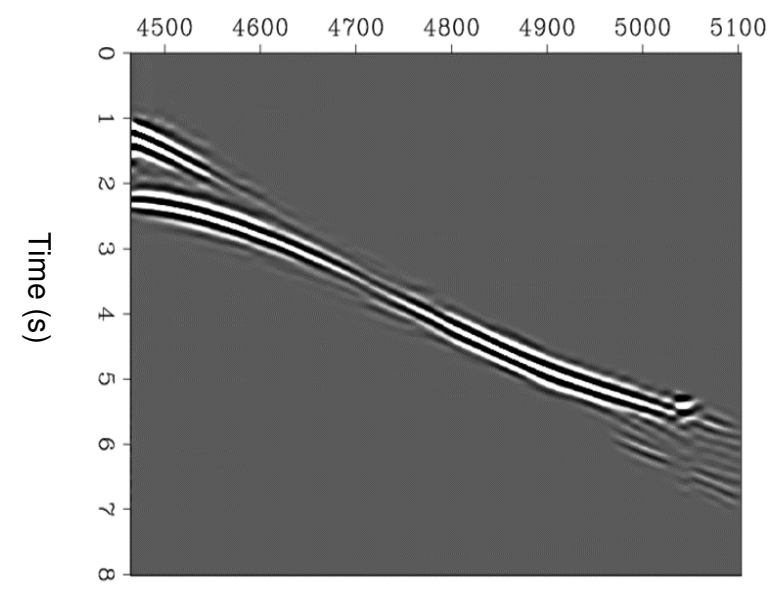

Figure 6 - Common-shot gather shown in figure 4, after application of a swell noise filter in CWT space. 


\section{References}

Akansu, A. N., Serdijn, W. A., \& Selesnick, I. W. (2010).

Emerging applications of wavelets: A review. Physical communication, 3(1), 1-18.

Virieux, J., \& Operto, S. (2009). An overview of fullwaveform inversion in exploration geophysics.

Geophysics, 74(6), WCC1-WCC26.

Yilmaz, Ö. (2001). Seismic data analysis: Processing, inversion, and interpretation of seismic data. Society of exploration geophysicists. 